\title{
Contents Vol. 49, 2004
}

\section{Neuropsychobiology}

No. 1

Biological Psychiatry

1 Association Study of Onset Age, Attempted Suicide, Aggressive Behavior, and Schizophrenia with a Serotonin 1B Receptor (A-161T) Genetic Polymorphism Hong, C.-J.; Pan, G.-M.; Tsai, S.-J. (Taipei)

5 Altered Habituation in the Auditory Cortex in a Subgroup of Depressed Patients by Functional Magnetic Resonance Imaging

Michael, N.; Ostermann, J. (Münster); Sörös, P. (Toronto); Schwindt, W.; Pfleiderer, B. (Münster)

10 Association Analysis of Brain-Derived Neurotrophic Factor Val66Met Polymorphisms with Alzheimer's Disease and Age of Onset

Tsai, S.-J.; Hong, C.-J.; Liu, H.-C.; Liu, T.-Y.; Hsu, L.-E. (Taipei); Lin, C.-H. (Kaohsiung)

13 Association Study of a Brain-Derived Neurotrophic Factor (BDNF) Val66Met Polymorphism and Personality Trait and Intelligence in Healthy Young Females

Tsai, S.-J.; Hong, C.-J. (Taipei); Yu, Y.W.-Y.; Chen, T.-J. (Kaohsiung)

17 The Influence of Parturition on the Level and Synthesis of Sulfated and Free Neurosteroids in Rats

Maayan, R.; Abou-Kaud, M. (Petah Tiqva); Strous, R.D. (Tel Aviv); Kaplan, B.; Fisch, B. (Petah Tiqva); Shinnar, N. (Tel Aviv); Weizman, A (Petah Tiqva)

24 Psychobiological Aspects of Somatoform Disorders: Contributions of Monoaminergic Transmitter Systems Rief, W. (Prien am Chiemsee/Marburg); Pilger, F.; Ihle, D. (Prien am Chiemsee); Verkerk, R.; Scharpe, S.; Maes, M. (Maastricht)

30 Self-Organizing Neural Network Analyses of Cardiac Data in Depression

Gaetz, M.; Iverson, G.L.; Rzempoluck, E.J.; Remick, R.; McLean, P.; Linden, W. (Vancouver)

38 Association between Major Depressive Disorder and the -1438A/G Polymorphism of the Serotonin 2A Receptor Gene

Choi, M.-J.; Lee, H.-J.; Lee, H.-J.; Ham, B.-J.; Cha, J.-H.; Ryu, S.-H.; Lee, M.-S. (Seoul)

Pharmacopsychiatry

42 Neurobiological and Psychological Correlates of Suicidal Attempts and Thoughts of Death in Patients with Major Depression

Fountoulakis, K.N.; Iacovides, A.; Fotiou, F.; Nimatoudis, J.; Bascialla, F.; Ioannidou, C.; Kaprinis, G. (Thessaloniki); Bech, P. (Hillerod)
No. 2

Biological Psychiatry

53 Paroxetine Decreases Respiratory Irregularity of Linear and Nonlinear Measures of Respiration in Patients with Panic Disorder. A Preliminary Report

Yeragani, V.K. (Detroit, Mich.); Rao, R. (Bangalore); Tancer, M.; Uhde, T. (Detroit, Mich.)

58 Endocrinological Effects of High-Dose Hypericum perforatum Extract WS 5570 in Healthy Subjects

Schüle, C.; Baghai, T.; Sauer, N.; Laakmann, G. (Munich)

64 Asperger Syndrome, Alexithymia and Perception of Sleep Tani, B.; Lindberg, N. (Helsinki); Joukamaa, M. (Tampere); Nieminen-von Wendt, T.; von Wendt, L.; Appelberg, B. (Helsinki); Rimón, R. (Lahti); Porkka-Heiskanen, T. (Helsinki)

71 High-Frequency Somatosensory Thalamocortical Oscillations and Psychopathology in Schizophrenia

Norra, C.; Waberski, T.D.; Kawohl, W.; Kunert, H.J.; Hock, D.; Gobbelé, R.; Buchner, H.; Hoff, P. (Aachen)

81 Platelet $\alpha_{2}$-Adrenoreceptors in Obsessive-Compulsive Disorder Marazziti, D.; Baroni, S.; Masala, I.; Giannaccini, G.; Mungai, F.; Di Nasso, E.; Cassano, G.B. (Pisa)

84 Pituitary-Thyroid State Correlates with Central Dopaminergic and Serotonergic Activity in Healthy Humans Strawn, J.R.; Ekhator, N.N.; D’Souza, B.B.; Geracioti, T.D., Jr. (Cincinnati, Ohio)

Biological Psychology/Pharmacopsychology

88 Attentional Modulation of Prepulse Inhibition: A New Startle Paradigm

Heekeren, K.; Meincke, U. (Aachen); Geyer, M.A. (La Jolla, Calif.); Gouzoulis-Mayfrank, E. (Aachen)

94 Augmentation of the Development of Behavioral Tolerance to Cannabinoid Administration through Pavlovian Conditioning Hill, M.N.; Gorzalka, B.B.; Choi, J.W. (Vancouver)

101 Whole Deletion of CYP2A6 Gene (CYP2A6*4C) and Smoking Behavior

Iwahashi, K.; Waga, C.; Takimoto, T. (Sagamihara)

Pharmacoelectroencephalography

105 Electroencephalographic Power and Coherence Analyses Suggest Altered Brain Function in Abstinent Male Heroin-Dependent Patients

Franken, I.H.A. (Den Haag/Rotterdam); Stam, C.J. (Amsterdam); Hendriks, V.M. (Den Haag); van den Brink, W. (Amsterdam)

\section{KARGER}

Fax +41613061234 E-Mail karger@karger.ch www.karger.com
(C) 2004 S. Karger AG, Base

Access to full text and tables of contents, including tentative ones for forthcoming issues: www.karger.com/nps_issues 
No. 3

Biological Psychiatry

111 Monocyte Chemoattractant Protein-1 Promoter -2518 Polymorphism May Have an Influence on Clinical Heterogeneity of Bipolar I Disorder in the Korean Population Pae, C.-U.; Kim, J.-J.; Yu, H.-S.; Lee, C.-U.; Lee, S.-J.; Jun, T.-Y.; Lee, C.; Paik, I.-H. (Seoul)

115 Regional Glucose Metabolism within Cortical Brodmann Areas in Healthy Individuals and Autistic Patients Hazlett, E.A.; Buchsbaum, M.S.; Hsieh, P.; Haznedar, M.M.; Platholi, J.; LiCalzi, E.M.; Cartwright, C.; Hollander, E. (New York, N.Y.)

126 Family-Based Association Study between the Monoamine Oxidase A Gene and Obesity: Implications for

Psychopharmacogenetic Studies

Camarena, B.; Santiago, H.; Aguilar, A.; Ruvinskis, E.; González-Barranco, J.; Nicolini, H. (México)

Pharmacopsychiatry

130 Tianeptine as a Slightly Effective Therapeutic Option for Attention-Deficit Hyperactivity Disorder Niederhofer, H. (Bolzano)

134 Brain Regions Activated during an Auditory Discrimination Task in Insomniac Postmenopausal Patients before and after Hormone Replacement Therapy: Low-Resolution Brain Electromagnetic Tomography Applied to Event-Related Potentials

Anderer, P.; Saletu, B.; Saletu-Zyhlarz, G.; Gruber, D.; Metka, M.; Huber, J. (Vienna); Pascual-Marqui, R.D. (Zurich)

Biological Psychology/Pharmacopsychology

154 Effects of Zolpidem and Zaleplon on Sleep, Respiratory Patterns and Performance at a Simulated Altitude of $\mathbf{4 , 0 0 0 ~ m ~}$ Beaumont, M.; Batéjat, D.; Coste, O.; van Beers, P.; Colas, A.; Clère, J.-M.; Piérard, C. (Brétigny-sur-Orge)

Pharmacoelectroencephalography

163 Global Approach to Multichannel Electroencephalogram Analysis for Diagnosis and Clinical Evaluation in Mild Alzheimer's Disease

Yoshimura, M.; Isotani, T.; Yagyu, T.; Irisawa, S.; Yoshida, T.; Sugiyama, M.; Minami, T.; Sugimoto, T.; Nobuhara, K., Okugawa, G.; Kinoshita, T. (Osaka)
No. 4

Biological Psychiatry

167 Cytochrome P-450 2D6* 10 C188T Polymorphism Is Associated with Antipsychotic-Induced Persistent Tardive Dyskinesia in Chinese Schizophrenic Patients

Liou, Y.-J. (Hualien/Taipei); Wang, Y.-C. (Hualien); Bai, Y.-M (Taipei); Chen, J.-Y.; Lai, I.-C. (Hualien)

174 Association between Norepinephrine Transporter Gene Polymorphism and Major Depression

Ryu, S.-H.; Lee, S.-H.; Lee, H.-J.; Cha, J.-H.; Ham, B.-J.; Han, C.-S.; Choi, M.-J.; Lee, M.-S. (Seoul)

178 Association Study of a Brain-Derived Neurotrophic Factor (Val66Met) Genetic Polymorphism and Panic Disorder Lam, P.; Cheng, C.-Y.; Hong, C.-J.; Tsai, S.-J. (Taipei)

182 No Association of a Functional Polymorphism in the Serotonin Transporter Gene Promoter and Anxiety-Related Personality Traits

Lang, U.E.; Bajbouj, M.; Wernicke, C.; Rommelspacher, H.; Danker-Hopfe, H.; Gallinat, J. (Berlin)

185 Banl Polymorphism of the Cytosolic Phospholipase $A_{2}$ Gene and Mood Disorders in the Korean Population Pae, C.-U.; Yu, H.-S.; Kim, J.-J.; Lee, C.-U.; Lee, S.-J.; Lee, K.-U.; Jun, T.-Y.; Paik, I.-H. (Seoul); Serretti, A. (Milan); Lee, C. (Seoul)

189 Altered 5-HT2A and 5-HT4 Postsynaptic Receptors and Their Intracellular Signalling Systems IP3 and cAMP in Brains from Depressed Violent Suicide Victims

Rosel, P.; Arranz, B.; Urretavizcaya, M.; Oros, M.; San, L.; Navarro, M.A. (Barcelona)

196 Association Study of a Functional Catechol-O-

Methyltransferase Genetic Polymorphism with Age of Onset, Cognitive Function, Symptomatology and Prognosis in Chronic Schizophrenia

Tsai, S.-J.; Hong, C.-J.; Liao, D.-L. (Taipei); Lai, I-C.; Liou, Y.-J. (Hualien)

Pharmacopsychiatry

201 Quetiapine Treatment for Behavioral and Psychological Symptoms in Patients with Senile Dementia of Alzheimer Type

Fujikawa, T.; Takahashi, T.; Kinoshita, A.; Kajiyama, H.; Kurata, A.; Yamashita, H.; Yamawaki, S. (Hiroshima)

205 Oxidative Damage and Schizophrenia:The Potential Benefit by Atypical Antipsychotics

Dakhale, G.; Khanzode, S.; Khanzode, S.; Saoji, A.; Khobragade, L.; Turankar, A. (Nagpur)

Biological Psychology/Pharmacopsychology

210 Declarative Memory Impairments following a Military Combat Course: Parallel Neuropsychological and Biochemical Investigations

Piérard, C. (Brétigny-sur-Orge); Béracochéa, D. (Talence); Pérès, M.; Jouanin, J.-C.; Liscia, P.; Satabin, P. (Brétigny-sur-Orge); Martin, S Testylier, G. (La Tronche); Guézennec, C.Y.; Beaumont, M. (Brétigny-sur-Orge)

Pharmacoelectroencephalography

218 Nonconvulsive Status epilepticus during Cephalosporin Therapy

Primavera, A.; Cocito, L.; Audenino, D. (Genova)

223 Author Index Vol. 49, 2004

225 Subject Index Vol. 49, 2004 